\title{
Bar $k$-Visibility Graphs: Bounds on the Number of Edges, Chromatic Number, and Thickness
}

\author{
Alice M. Dean ${ }^{1}$, William Evans ${ }^{2}$, Ellen Gethner ${ }^{3}$, Joshua D. Laison ${ }^{4}$, \\ Mohammad Ali Safari ${ }^{5}$, and William T. Trotter ${ }^{6}$ \\ 1 Department of Mathematics and Computer Science, Skidmore College \\ adean@skidmore.edu \\ 2 Department of Computer Science, University of British Columbia \\ will@cs.ubc.ca \\ 3 Department of Computer Science and Engineering, \\ University of Colorado at Denver \\ ellen.gethner@cudenver.edu \\ 4 Department of Mathematics and Computer Science, Colorado College \\ jlaison@coloradocollege.edu \\ 5 Department of Computer Science, University of British Columbia \\ safari@cs.ubc.ca \\ ${ }^{6}$ Department of Mathematics, Georgia Institute of Technology \\ trotter@math.gatech.edu
}

\begin{abstract}
Let $S$ be a set of horizontal line segments, or bars, in the plane. We say that $G$ is a bar visibility graph, and $S$ its bar visibility representation, if there exists a one-to-one correspondence between vertices of $G$ and bars in $S$, such that there is an edge between two vertices in $G$ if and only if there exists an unobstructed vertical line of sight between their corresponding bars. If bars are allowed to see through each other, the graphs representable in this way are precisely the interval graphs. We consider representations in which bars are allowed to see through at most $k$ other bars. Since all bar visibility graphs are planar, we seek measurements of closeness to planarity for bar $k$-visibility graphs. We obtain an upper bound on the number of edges in a bar $k$-visibility graph. As a consequence, we obtain an upper bound of 12 on the chromatic number of bar 1-visibility graphs, and a tight upper bound of 8 on the size of the largest complete bar 1-visibility graph. We conjecture that bar 1-visibility graphs have thickness at most 2 .
\end{abstract}

\section{Introduction}

Recent attention has been drawn to a variety of generalizations of bar visibility graphs $2,3,6,6,5,7,8,11,12,14,15$. In this note, we report on a new generalization of bar visibility graphs called bar $k$-visibility graphs, and discuss some of their properties; complete details can be found in [4. In what follows, we use the standard graph theory terminology found in 9, 17.

Let $S$ be a set of disjoint horizontal line segments, or bars, in the plane. We say that a graph $G$ is a bar visibility graph, and $S$ a bar visibility representation 


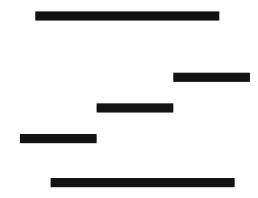

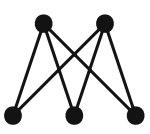

G

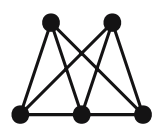

$\mathrm{H}$

Fig. 1. The bar visibility representation shown is an $\varepsilon$-visibility representation of $G$ and a strong visibility representation of $H$

of $G$, if there exists a one-to-one correspondence between vertices of $G$ and bars in $S$, such that there is an edge between two vertices $x$ and $y$ in $G$ if and only if there exists a vertical line segment $L$, called a line of sight, whose endpoints are contained in $X$ and $Y$, respectively, and which does not intersect any other bar in $S$. [1, 12, 13, 18.

If each line of sight is required to be a rectangle of positive width, then $S$ is an $\varepsilon$-visibility representation of $G$, and when each line of sight is a line segment, then $S$ is a strong visibility representation of $G$ [16]. In general, these definitions are not equivalent; $K_{2,3}$ admits an $\varepsilon$-visibility representation but not a strong visibility representation, as shown in Figure 1

Given a set of bars $S$ in the plane, suppose that an endpoint of a bar $B$ and an endpoint of a bar $C$ in $S$ have the same $x$-coordinate. We elongate one of these two bars so that their endpoints have distinct $x$-coordinates. If $S$ is a strong visibility representation of a graph $G$, then we may perform this elongation so that $S$ is still a strong visibility representation of $G$. If $S$ is an $\varepsilon$-visibility representation of $G$, then we may perform this elongation so that $S$ is an $\varepsilon$-visibility representation of a new graph $H$ with $G \subseteq H$. Since we are interested in the maximum number of edges obtainable in a representation, we may consider the graph $H$ instead of the graph $G$. Repeating this process yields a set of bars with pairwise distinct endpoint $x$-coordinates. For the remainder of this paper, we assume that all bar visibility representations are of this form.

If a set of bars $S$ has all endpoint $x$-coordinates distinct, the graphs $G$ and $H$ that have $S$ as a strong bar visibility representation and an $\varepsilon$-visibility representation, respectively, are isomorphic. Hence without loss of generality, for the remainder of the paper, all bar visibility representations are strong bar visibility representations.

By contrast, suppose that $S$ is a set of closed intervals on the real line. The graph $G$ is called an interval graph and $S$ an interval representation of $G$ if there exists a one-to-one correspondence between vertices of $G$ and intervals in $S$, such that $x$ and $y$ are adjacent in $G$ if and only if their corresponding intervals intersect. Suppose we call a set $S$ of horizontal bars in the plane an $x$-ray-visibility representation if we allow sight lines to intersect arbitrarily many bars in $S$. Then we can easily transform an $x$-ray-visibility representation into an interval representation by vertically translating the bars in $S$, and vice-versa. Therefore $G$ is an $x$-ray-visibility graph if and only if $G$ is an interval graph.

Motivated by this correspondence, we define a bar $k$-visibility graph to be a graph with a bar visibility representation in which a sight line between bars 
$X$ and $Y$ intersects at most $k$ additional bars. As a first step on the road to a characterization of bar $k$-visibility graphs, since all bar visibility graphs are planar, we seek measurements of closeness to planarity for bar $k$-visibility graphs.

\section{An Edge Bound for Bar 1-Visibility Graphs}

Suppose $G$ is a graph with $n$ vertices, and $S$ is a bar 1-visibility representation of $G$. Since we consider $S$ to be a strong visibility representation of $G$, without loss of generality, we may assume that all endpoints of all bars in $S$ have distinct $x$-coordinates, and all bars in $S$ have distinct $y$-coordinates.

It will be convenient to use four different labeling systems for the bars in $S$. Label the bars $1_{l}, 2_{l}, \ldots, n_{l}$ in increasing order of the $x$-coordinate of their left endpoint. Label them $1_{r}, 2_{r}, \ldots, n_{r}$ in decreasing order of the $x$-coordinate of their right endpoint. Label them $1_{b}, 2_{b}, \ldots, n_{b}$ in increasing order of their $y$-coordinate. Finally, label them $1_{t}, 2_{t}, \ldots, n_{t}$ in decreasing order of their $y$ coordinate. So the bar $1_{l}$ has leftmost left endpoint, the bar $1_{r}$ has rightmost right endpoint, the bar $1_{b}=n_{t}$ is bottommost in the representation, and the bar $1_{t}=n_{b}$ is topmost in the representation. We use this notation for the remainder of the paper.

Remark 1. Suppose $S$ is a bar $k$-visibility representation of a graph $G$ with $n$ vertices. We elongate the top and bottom bars of $S$ to obtain a new bar $k$ visibility representation $S^{\prime}$ of a new graph $G^{\prime}$, with the additional property that $1_{t}=1_{r}=1_{l}$ and $1_{b}=2_{r}=2_{l}$ in $S^{\prime}$. The graph $G^{\prime}$ has $n$ vertices and contains $G$ as a subgraph. We may therefore assume that every edge-maximal bar $k$-visibility graph has such a bar $k$-visibility representation.

Lemma 1. If $G$ is a bar 1-visibility graph with $n \geq 4$ vertices, then $G$ has at most $6 n-17$ edges.

Proof. Suppose $G$ is a graph with $n$ vertices, and $S$ is a bar 1-visibility representation of $G$. We define the following correspondence between bars in $S$ and edges of $G$. Let $U$ be the bar in $S$ associated with vertex $u$. For every edge $\{u, v\}$ in $G$, let $\ell(\{u, v\})$ be the vertical line segment from a point in $U$ to a point in $V$ whose $x$-coordinate is the infimum of $x$ coordinates of lines of sight between $U$ and $V$. An edge $\{u, v\}$ is called a left edge of $U$ (respectively $V$ ) if $\ell(\{u, v\}$ ) contains the left endpoint of $U$ (respectively $V)$. If $\ell(\{u, v\})$ contains neither $U$ nor $V$ 's left endpoint then it must contain the right endpoint of some bar $B$ (that blocks the 1-visibility of $U$ from $V$ from that point on). In this case, we call $\{u, v\}$ a right edge of $B$. Note that the right edges of $B$ are not incident to the vertex $b$ of $G$ corresponding to the bar $B$. Each bar $B$ can have at most 4 left edges (two to bars above $B$ in $S$ and two to bars below $B$ in $S$ ) and at most 2 right edges, as shown in Figure 2 .

Counting both left and right edges, each bar in $S$ is associated with at most 6 edges. So there are at most $6 n$ edges in $G$. However, the bars $1_{l}, 2_{l}, 3_{l}$, and $4_{l}$ have at most $0,1,2$, and 3 left edges, respectively. Similarly, the bars $1_{r}, 2_{r}, 3_{r}$, 


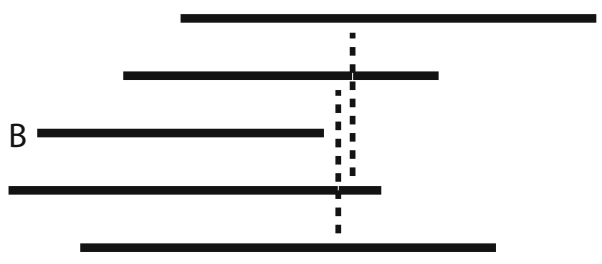

Fig. 2. The two right edges associated to bar $B$

and $4_{r}$ have at most $0,0,0$, and 1 right edges, respectively. Therefore there are at most $4 n-10$ left edges and at most $2 n-7$ right edges, for a total of at most $6 n-17$ edges in $G$.

Theorem 1. If $G$ is a bar 1-visibility graph with $n \geq 5$ vertices, then $G$ has at most $6 n-20$ edges.

Proof. We improve the bound given in Lemma 1 by using a slightly more sophisticated technique. We follow the notation of Lemma 1.

By Remark 1, the edge $\left\{1_{t}, 1_{b}\right\}$ will always be a left edge. Since the edge associated with the right endpoint of the bar $4_{r}$ can only be this edge, the bar $4_{r}$ must have 0 right edges. So there are at most $2 n-8$ right edges in $G$, and $6 n-18$ edges in total. If $G$ has exactly $6 n-18$ edges, then bars $1_{l}, 2_{l}, 3_{l}$, and $4_{l}$ must have at least $0,1,2$, and 2 left edges, respectively.

Suppose that bar $4_{l}$ has only two left edges. Then it does not have a line of sight to bar $3_{l}$, which can happen only if $3_{l}$ ends before $4_{l}$ begins. Then $3_{l}=n_{r}$, and $3_{l}$ has 0 right edges. Therefore $G$ has at most $6 n-20$ edges. The only remaining possibility is that bar $4_{l}$ has exactly three left edges.

If $S$ had at most $4 n-12$ left edges, then $S$ would have at most $6 n-20$ edges in total. The remaining possibilities are that $S$ has either $4 n-11$ or $4 n-10$ left edges. Since $1_{l}, 2_{l}, 3_{l}$, and $4_{l}$ have exactly $0,1,2$, and 3 left edges, respectively, all other bars in $S$ must have exactly four left edges, except perhaps for one bar $i_{l}$, which may have three left edges. By the same argument, since $1_{r}, 2_{r}, 3_{r}$, and $4_{r}$ have no right edges, every additional bar must have exactly two right edges, except one additional bar, which may have only one.

Consider the four edges $e_{1}=\left\{1_{t}, 1_{b}\right\}, e_{2}=\left\{1_{t}, 2_{b}\right\}, e_{3}=\left\{2_{t}, 1_{b}\right\}$, and $e_{4}=$ $\left\{2_{t}, 2_{b}\right\}$. If $i_{l}=2_{b}$, then the edges $e_{1}$ and $e_{3}$ are left edges, but the edges $e_{2}$ and $e_{4}$ may not be. If $i_{l}=2_{t}$, then the edges $e_{1}$ and $e_{2}$ are left edges, but the edges $e_{3}$ and $e_{4}$ may not be. If $i_{l}$ is neither of these bars, then all four of these edges are left edges.

Since the bars $2_{t}$ and $2_{b}$ have at most one right edge each, one of them must be bar $3_{r}$ or bar $4_{r}$. Without loss of generality, assume that bar $2_{t}$ is either bar $3_{r}$ or bar $4_{r}$. So in the order of the bars $1_{r}$ through $5_{r}$ given by increasing $y$-coordinate, the bar $5_{r}$ must appear either second or third. Figure 3 shows the four possibilities that may occur.

In each of the four cases shown, and for each of the three possibilities for the bar $i_{l}$, one can check that $5_{r}$ has at most one right edge. So the remaining bars must all have exactly two right edges. Therefore the bars $2_{t}$ and $2_{b}$ must be two 

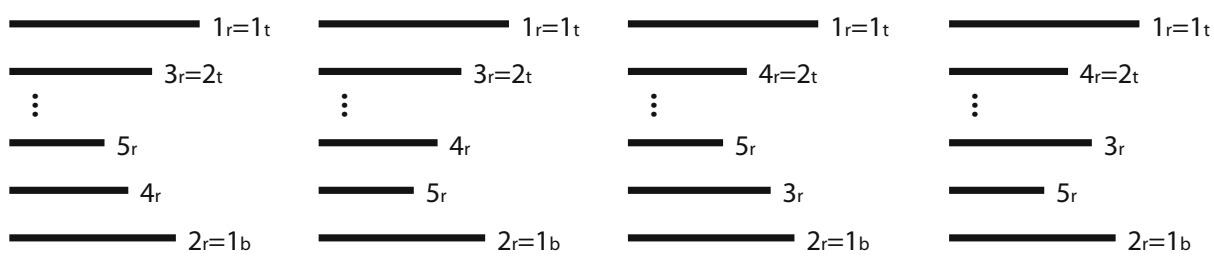

Fig. 3. The four possible arrangements of bars $1_{r}, 2_{r}, 3_{r}, 4_{l}$, and $5_{r}$

of the three bars $3_{r}, 4_{r}$, and $5_{r}$. But this implies that any right edge associated to $5_{r}$ must be between a pair of the bars $1_{t}, 2_{t}, 1_{b}$, and $2_{b}$. Therefore $5_{r}$ must have no right edges, and $G$ has at most $6 n-20$ edges in total.

Corollary 2. The graph $K_{9}$ is not a bar 1-visibility graph.

Proof. Any bar 1-visibility graph with 9 vertices has at most 34 edges, whereas $K_{9}$ has 36 edges.

Corollary 3. If $G$ is a bar 1-visibility graph, then $\chi(G) \leq 12$.

Proof. We proceed by induction. Assume that all bar 1-visibility graphs with $n-1$ vertices have $\chi \leq 12$, and suppose that $G$ is a bar 1 -visibility graph with $n$ vertices. By Theorem 1, $\sum_{v \in V(G)} \operatorname{deg}(v)<12 n$, so the average degree of a vertex in $G$ is strictly less than 12 . Then there must exist a vertex $v$ in $G$ of degree at most 11. We consider the graph $G-v$. Although this graph may not be a bar 1-visibility graph, it is a subgraph of the graph $G^{\prime}$ with bar 1-visibility representation obtained from a representation of $G$ by deleting the bar corresponding to $v$. Therefore the edge bound in Theorem 1 still applies to $H$. By the induction hypothesis, we may color the vertices of $H$ with 12 colors, replace $v$, and color $v$ with a color not used on its neighbors.

Corollary 4. There are thickness-2 graphs with $n$ vertices that are not bar 1 visibility graphs for all $n \geq 15$.

Proof. Note that there are no thickness- 2 graphs with $n$ vertices and more than $6 n-12$ edges, since if $G$ has thickness 2 then $G$ is the union of two planar graphs, each of which have at most $3 n-6$ edges. Consider the graph $G=C_{3} \otimes C_{5}$ formed by replacing each vertex in $C_{5}$ with $C_{3}$ and taking the join of neighboring $C_{3}$ 's. $G$ has 15 vertices and $6 \cdot 15-12=78$ edges. Since $G$ is the union of the two planar graphs shown in Figure 4, $G$ has thickness 2 .

Let $G_{15}=G$ and suppose $L_{1}$ and $L_{2}$ are the two plane layers of $G_{15}$. Let $\{a, b, c\}$ be a face in $L_{1}$ and $\{d, e, f\}$ be a face in $L_{2}$ such that $\{a, b, c\} \cap\{d, e, f\}=$ $\varnothing$. Add a new vertex $v$ to $G_{15}$ adjacent to $\{a, b, c\}$ in $L_{1}$ and $\{d, e, f\}$ in $L_{2}$; define the new graph to be $G_{16}$. The graph $G_{16}$ has 16 vertices and $6 \cdot 16-12$ edges, and thickness 2 . Following the same procedure, inductively we construct an infinite family of graphs $G_{n}$ such that for all $n \geq 15, G_{n}$ has $n$ vertices and $6 n-12$ edges, and thickness 2 . Therefore none of these graphs can be a bar 1-visibility graph by Theorem 1 . 

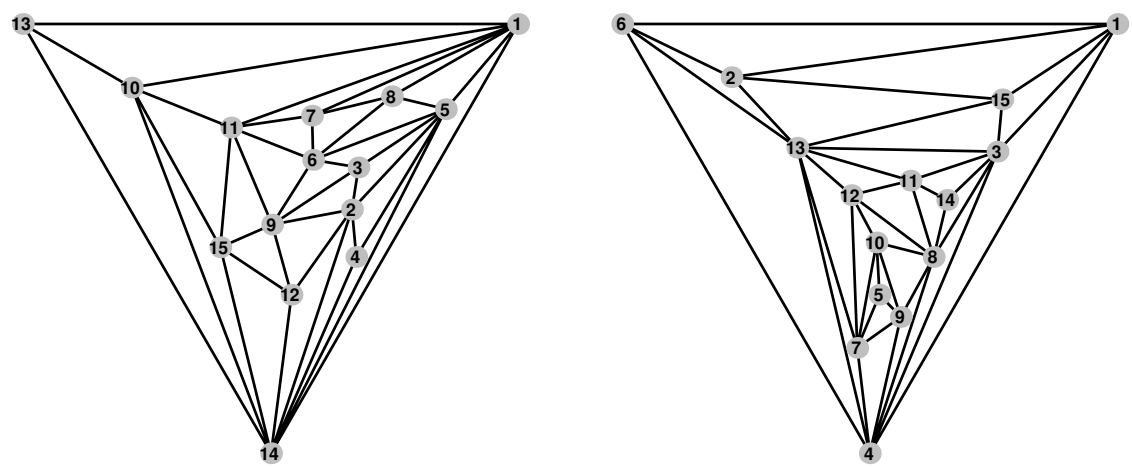

Fig. 4. Two planar graphs whose union is not a bar 1-visibility graph

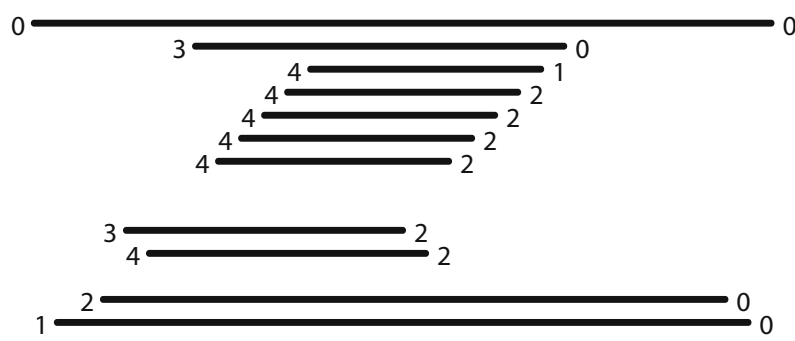

Fig. 5. A bar 1-visibility representation with $6 n-20$ edges

Note that the graphs $\left\{G_{n}\right\}$ given in the proof of Corollary 4 have the largest possible number of edges while having thickness 2 .

Theorem 5. There exist bar 1-visibility graphs with $6 n-20$ edges, $n \geq 5$.

Proof. The graph with representation shown in Figure 5 is a bar 1-visibility graph with $6 n-20$ edges. For ease of counting, the left and right endpoints of bars in this representation are labeled with the number of left and right edges associated to each bar. Note that this representation has $4 n-11$ left edges and $2 n-9$ right edges. Although $n=11$ in this representation, more bars can easily be deleted to create a representation with as few as 5 bars, or added to create a representation with arbitrarily many bars. For the values $n=5$ through 8 , this representation yields a complete graph.

Corollary 6. The graph $K_{8}$ is a bar 1-visibility graph.

Proof. Take only eight bars in the representation shown in Figure 5 .

By Corollary [6] if $G$ is a bar 1-visibility graph, then $\chi(G)$ may be 8 . No bar 1 -visibility graph is known with chromatic number 9 . The standard example of a graph with chromatic number 9 but clique number smaller than 9 is the Sulanke graph $K_{6} \vee C_{5}$ [17, which is not a bar 1-visibility graph since it has 11 vertices and 50 edges. 


\section{Edge Bounds on Bar $k$-Visibility Graphs}

The following theorem generalizes Lemma 1 for $k>1$. The proof is entirely analogous to the proof of Lemma 1, and can be found in [4.

Theorem 7. If $G$ is a bar $k$-visibility graph with $n \geq 2 k+2$ vertices, then $G$ has at most $(k+1)\left(3 n-\frac{7}{2} k-5\right)$ edges.

Theorem 8. There exist bar $k$-visibility graphs with $n$ vertices and $(k+1)(3 n-$ $4 k-6)$ edges for $k \geq 0$ and $n \geq 3 k+3$.

Proof. Figure 6 shows a bar $k$-visibility representation of a graph with $n$ vertices and $(k+1)(3 n-4 k-6)$ edges. As in Figure [5] the left and right endpoints of bars in this representation are labeled with the number of left and right edges associated to each bar. Although $n=4 k+4$ in this representation, more bars can easily be deleted to create a representation with as few as $3 k+3$ bars, or added to create a representation with arbitrarily many bars.

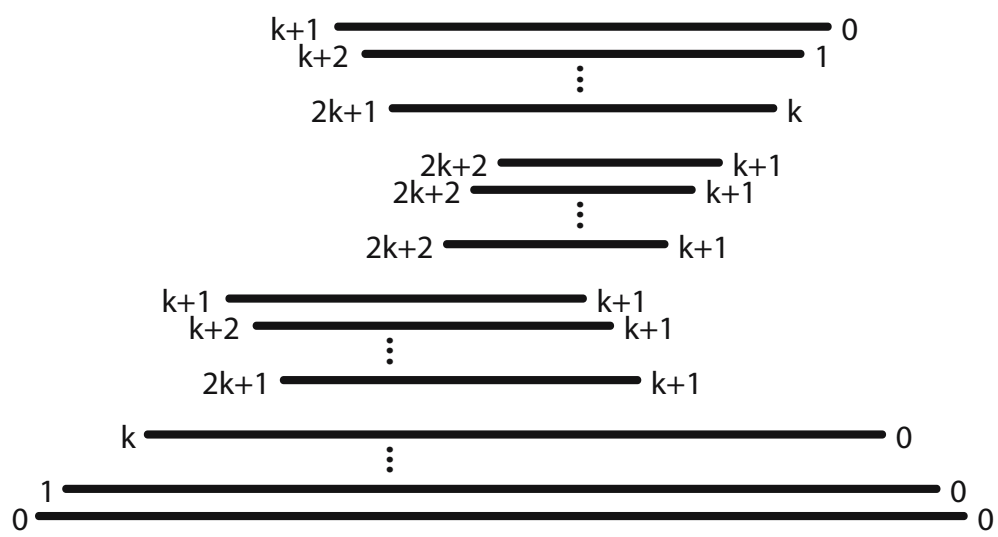

Fig. 6. A bar $k$-visibility graph with $n$ vertices and $(k+1)(3 n-4 k-6)$ edges

Note that Theorem 8 gives the largest number of edges in a bar $k$-visibility graph for $k=0,1$. We believe that this is the case for larger $k$ as well. We state this as a conjecture.

Conjecture 1 . If $G$ is a bar $k$-visibility graph, then $G$ has at most $(k+1)(3 n-$ $4 k-6)$ edges.

The following theorem is a corollary of Theorem 7 .

Theorem 9. $K_{5 k+5}$ is not a bar k-visibility graph.

Proof. By way of contradiction, suppose that $G$ is a graph with $n=5 k+5$ vertices. Then by Theorem 7 . $G$ has at most $(k+1)\left(3(5 k+5)-\frac{7}{2} k-5\right)=$ $\frac{23}{2} k^{2}+\frac{43}{2} k+10$ edges. However, $K_{5 k+5}$ has $\left(\begin{array}{c}5 k+5 \\ 2\end{array}\right)=\frac{25}{2} k^{2}+\frac{45}{2} k+10$ edges. 
Note that if Conjecture 1 is true, we immediately obtain the following conjecture as a corollary.

Conjecture 2. $K_{4 k+4}$ is the largest complete bar $k$-visibility graph.

Proof (Assuming Conjecture 1). Figure 6 shows a bar $k$-visibility representation of $K_{4 k+4}$. Conversely, suppose that $G$ is a graph with $n=4 k+5$ vertices. Then by Conjecture 1, $G$ has at most $(k+1)(3(4 k+5)-4 k-6)=8 k^{2}+17 k+9$ edges. However, $K_{4 k+5}$ has $\left(\begin{array}{c}4 k+5 \\ 2\end{array}\right)=8 k^{2}+18 k+10$ edges.

Conjecture 1 is not required to prove Conjecture 2 when $k=0$ or 1 ; we have already proved these cases in the previous section. Note also that the graph $K_{4 k+4}$ exactly achieves the bound given by Conjecture 11. So if this conjecture is correct, the family of complete graphs $K_{4 k+4}$ is an example of a family of edge-maximal bar $k$-visibility graphs.

\section{Thickness of Bar $k$-Visibility Graphs}

By Corollary 6, $K_{8}$ is a bar 1-visibility graph, and thus there are non-planar bar 1-visibility graphs. Motivated by the fact that all bar 0-visibility graphs are planar [10], we are interested in measuring the closeness to planarity of bar 1-visibility graphs. The thickness $\Theta(G)$ of a graph $G$ is the minimum number of planar graphs whose union is $G$. $K_{8}$ has thickness 2 [12, so there exist bar 1-visibility graphs with thickness 2 . Conversely, the following theorem from [4] gives an upper bound for the thickness of a bar 1-visibility graph.

Suppose $G$ is a bar 1-visibility graph, and $S$ is a bar 1-visibility representation of $G$. We define the underlying bar visibility graph $G_{0}$ of $S$ to be the graph with bar visibility representation $S$. The following theorem relates the thickness of $G$ to the chromatic number of $G_{0}$.

Theorem 10. If $G$ is a bar 1-visibility graph and $G_{0}$ an underlying bar visibility graph of $G$, then $\Theta(G) \leq \chi\left(G_{0}\right)$. In particular, the thickness of any bar 1visibility graph is at most four.

We conjecture that bar 1-visibility graphs have thickness no greater than 2 . More generally, we know that the thickness of a bar $k$-visibility graph is bounded by some function of $k$ [4. The smallest such function of $k$ is still open.

\section{Future Work}

We close with a list of open problems inspired by the results of this note.

1. What is the largest number of edges in a bar 2-visibility graph with $n$ vertices?

2. What is the largest number of edges in a bar $k$-visibility graph with $n$ vertices? 
3. Are there bar 1-visibility graphs with thickness 3 ?

4. More generally, what is the largest thickness of a bar $k$-visibility graph? Is it $k+1$ ?

5. Are there bar 1-visibility graphs with chromatic number 9 ?

6. More generally, what is the largest chromatic number of a bar $k$-visibility graph?

7. What is the largest crossing number of a bar $k$-visibility graph?

8. What is the largest genus of a bar $k$-visibility graph?

9 . What is a complete characterization of bar $k$-visibility graphs?

10. Is there an efficient recognition algorithm for bar $k$-visibility graphs?

11. Rectangle visibility graphs are defined in [7,8,15. Generalize the results of this note to rectangle visibility graphs.

12. Arc- and circle-visibility graphs are defined in [11. Generalize the results of this note to arc- and circle-visibility graphs.

\section{References}

1. T. Andreae. Some results on visibility graphs. Discrete Appl. Math., 40(1):5-17, 1992. Combinatorial methods in VLSI.

2. P. Bose, A. Dean, J. Hutchinson, and T. Shermer. On rectangle visibility graphs. In Lecture Notes in Computer Science 1190: Graph Drawing, pages 25-44. SpringerVerlag, 1997.

3. G. Chen, J. P. Hutchinson, K. Keating, and J. Shen. Characterizations of $1, k$-bar visibility trees. In preparation, 2005.

4. A. Dean, W. Evans, E. Gethner, J. D. Laison, M. A. Safari, and W. T. Trotter. Bar $k$-visibilty graphs. Submitted, 2005.

5. A. M. Dean, E. Gethner, and J. P. Hutchinson. A characterization of triangulated polygons that are unit bar-visibility graphs. In preparation, 2005.

6. A. M. Dean, E. Gethner, and J. P. Hutchinson. Unit bar-visibility layouts of triangulated polygons: Extended abstract. In J. Pach, editor, Lecture Notes in Computer Science 3383: Graph Drawing 2004, pages 111-121, Berlin, 2005. Springer-Verlag.

7. A. M. Dean and J. P. Hutchinson. Rectangle-visibility representations of bipartite graphs. Discrete Appl. Math., 75(1):9-25, 1997.

8. A. M. Dean and J. P. Hutchinson. Rectangle-visibility layouts of unions and products of trees. J. Graph Algorithms Appl., 2:no. 8, 21 pp. (electronic), 1998.

9. G. Di Battista, P. Eades, R. Tamassia, and I. G. Tollis. Graph Drawing. Prentice Hall Inc., Upper Saddle River, NJ, 1999.

10. M. R. Garey, D. S. Johnson, and H. C. So. An application of graph coloring to printed circuit testing. IEEE Trans. Circuits and Systems, CAS-23(10):591-599, 1976.

11. J. P. Hutchinson. Arc- and circle-visibility graphs. Australas. J. Combin., 25:241262, 2002.

12. J. P. Hutchinson, T. Shermer, and A. Vince. On representations of some thicknesstwo graphs. Computational Geometry, 13:161-171, 1999.

13. P. Rosenstiehl and R. E. Tarjan. Rectilinear planar layouts and bipolar orientations of planar graphs. Discrete Comput. Geom., 1(4):343-353, 1986.

14. T. Shermer. On rectangle visibility graphs III. External visibility and complexity. In Proc. 8th Canad. Conf. on Comp. Geom., pages 234-239, 1996. 
15. I. Streinu and S. Whitesides. Rectangle visibility graphs: characterization, construction, and compaction. In STACS 2003, volume 2607 of Lecture Notes in Computer Science, pages 26-37. Springer, Berlin, 2003.

16. R. Tamassia and I. G. Tollis. A unified approach to visibility representations of planar graphs. Discrete Comput. Geom., 1(4):321-341, 1986.

17. D. B. West. Introduction to Graph Theory, 2E. Prentice Hall Inc., Upper Saddle River, NJ, 2001.

18. S. K. Wismath. Characterizing bar line-of-sight graphs. In Proceedings of the First Symposium of Computational Geometry, pages 147-152. ACM, 1985. 